\title{
2
}

\section{The comparative semantics of verbs of 'opening': West Africa vs Oceania}

\author{
Felix K. Ameka and Deborah Hill
}

\section{Introduction}

Separating things into parts or constituents using different means is an everyday activity that humans everywhere undertake. The way in which humans categorise such events and label them in verbs varies considerably across languages and cultures. In a cross-linguistic study of the categorisation of separation events, Majid et al. (2007: 147) note that separation events such as opening, taking apart and peeling, which involve minimal destruction of the affected object and are mostly reversible, were distinguished from those involving cutting and breaking, which involve significant material destruction accompanied by non-reversible change in the integrity of the object.

'Opening' events are thus a subspecies of separation events. Many languages partition the 'opening' events in English among a number of different verbs, but the criteria used for this differ strikingly from one language to another. An English-like category of opening events does not seem to be inevitable to human cognition (Bowerman 2005: 229). For instance, Korean has about six verbs that cover the semantic range of opening events in English. As Bowerman put it: 
The conceptual glue that unifies for example 'opening the mouth', 'opening an envelope', and 'opening a book' for speakers of English seems to be missing [in Korean FKA \& DH] and the domain is parceled out among a number of crosscutting categories that emphasise different aspects of events. (Bowerman 2005: 228)

This chapter compares the way two languages, Ewe, a Kwa language of West Africa, and Longgu, an Oceanic language of Solomon Islands, carve up the semantic space of 'opening' and provides semantic explications for a small number of verbs in each language. ${ }^{1}$

In morpho-syntactic terms, Ewe and Longgu share some similarities and they both deploy serial verb constructions (SVCs). They both also use reduplication to express various meanings. There are some differences too. Longgu is a head marking language where arguments are marked on the verb following an SVO (subject-verb-object) pattern. However, constituent order in the clause is typically VS/VOA (verb-subject/verbobject-transitive subject, Hill 2016a). The basic constituent order in Ewe by contrast is SV/AVO (subject-verb/transitive subject-verb-object). In addition, Longgu has verb transitivising suffixes, as we shall see. Ewe, on the other hand, is an isolating language with agglutinative features. It does not use verb derivational morphology to express transitivity and other valency changing processes. Rather, the verbs participate in multiple argument alternations. Ewe is also a tone language.

The lexicon of complex physical activities in different languages reveals differences related to culture, as well as similarities between cultures (Goddard and Wierzbicka 2009). This is reflected in verbs in the domain of 'carrying' (Hill 2016b; Ameka 2017) as well as in separation events of cutting and breaking (Ameka and Essegbey 2007; Goddard and Wierzbicka 2009). We shall demonstrate that the verbs of opening also

1 Indulging in this comparative semantics of two languages spoken on continents apart allows us to reflect on and engage with the teaching and mentorship and friendship we have experienced with Anna for about three and a half decades. The authors first met in one of Anna's classes in March 1984 and have been on the quest for meaning with her since. We have enjoyed many debates with Anna not only in class but also during the first Semantics workshop in Adelaide in 1986 and subsequently. We are delighted to honour Anna on the occasion of her anniversary comparing verbs of opening in Ewe, the native language of the first author, and the first African language to have been studied within the Natural Semantic Metalanguage (NSM) framework, and Longgu, the field language specialisation of the second author who is a first-generation practitioner of NSM.

For us, the power of NSM semantic representations is the way in which it helps the analyst to allow the language to speak. Moreover, the semantic explications of signs in different languages can be easily compared and the similarities and differences become transparent even to the casual reader. 
show this diversity and cultural embeddedness. Examples of culturally relevant verbs of opening in Longgu are the verbs suvulia 'open the eye of a coconut with an instrument (e.g. a stick or knife)'; and tuaa 'open the side of a bamboo by putting a hole in it (with a knife)'. Objects that are opened with instruments tend to be lexically specific in Longgu (i.e. the verb refers to opening one object in a particular way). Where Longgu has at least 10 verbs that express opening events, Ewe has three. Opening events such as 'peel something' are expressed by a specific verb in Longgu (pagea 'peel something (e.g. banana)'), whereas in Ewe one of three verbs expressing opening $\left(k l \tilde{e}^{\prime}\right)$, is also used to describe peeling.

While Ewe and Longgu carve up the semantic space of opening events differently, there are also shared properties of objects of physical actions of opening. These common properties reflect some of the properties of objects for physical action of opening events in English identified by Bowerman (2005) and include the six senses of English 'open' proposed by Levison (1993). Bowerman suggested that for English, 'openable' objects can be described as: (a) a unitary object, although it may have parts such as a pot with a lid; (b) an object that separates along predetermined lines not unpredictably (hence actions of opening are usually reversible; objects that can be opened can also be closed); and (c) separation affords access to something (e.g. a content, an interior space or a previously concealed part of the object with which you can do something). These characteristics suggest, prototypically, a container (as exemplified by the pot referred to). Levison (1993) also includes 'containers' (e.g. open the can/bottle/soda; open the house) and 'conduits' (channels and paths) as two of six suggested senses of English 'open'. Both Ewe and Longgu have verbs ( $v u$ and tavangia) to refer to opening containers and conduits. However, when these verbs are used to describe the opening of 'channels' or 'paths', the languages differ in the range of objects that can be referred to; for example, while Ewe $v u$ and Longgu tavangia can be used to refer to opening or turning on a radio, only Longgu tavangia is used to refer to turning on or opening a lamp or light.

For the interpretation of linguistic signs and utterances, we assume following Wilkins and Hill (1995) that there are three levels of meaning (see also e.g. Kecskes 2008; Levinson 2000). The first level, Semantics 1, concerns the stable, context-independent meaning values of signs. These are the structured ideas about signs (lexical items, constructions, gestures, prosodic patterns etc.) that speakers share which are stored in the mind. It is these stable, intersubjective structured ideas about the 
verbs in the two languages that we strive to represent in the semantic explications proposed. Combinatorial rules generate an output, the literal meaning. This feeds into level 2, which is a kind of fill-in, or a filter box (Pragmatics). At this level, the literal meaning of an utterance interacts with neo-Gricean generalised Conversational Implicatures (Levinson 2000), cultural scripts (e.g. Goddard and Wierzbicka 2004), semantic frames (e.g. Fillmore and Atkins 1992) and all kinds of world and encyclopedic knowledge. These processes of enrichment and filtering lead to the online contextual interpretation of utterances (for both speaker and hearer), the third level (Semantics 2) (Wilkins and Hill 1995). These contextual interpretations may be cycled back into Semantics 1, where they become more stable meaning values of signs. This is the way in which meaning change takes place.

The organisation of the chapter is as follows: we first introduce verbs of opening in Ewe (Section 2). Section 3 presents an overview of the Longgu verbs and discusses two that share semantic similarities with the Ewe verbs. In Section 4, we draw out parallels and differences between the two languages and discuss them in the context of the key characteristics of openable objects in English, suggested by Bowerman (2005), and propose explications for them based on the Natural Semantic Metalangauge (NSM) principles following the semantic template for verbs proposed by Goddard and Wierzbicka (2016). The explications couched in semantic primes allow for easy comparison of the meanings across the two languages.

\section{Opening events in Ewe}

The verbs in Ewe are ke 'open, end, spread out, etc.', klế 'open, peel, remove outer covering; shine', and $v u$ 'open'. The three verbs in Ewe that carve up the space of opening events have distinct semantics. Nevertheless, they may apply to the same entities revealing different construals of the opening events. Thus each of the verbs can take the body parts with two parts such as $\eta k u$ ' eye', nu 'mouth', glä 'jaw' as object with different nuances of interpretation. Thus ke nu'open mouth' implies that the two lips move apart because someone wants it; klé $n u$ 'open mouth wide' implies that the lips come apart as someone wants it, because of this one can see inside the mouth' and $v u$ nu 'open mouth' describes a situation where someone consciously and purposefully opens the mouth (e.g. to talk). It entails that 
it was done purposefully. In the next subsection we summarise the uses and interpretations of $k e$ 'open' as presented in Ameka (2019). We then turn attention to the other Ewe verb $v u$ 'open'.

\subsection{The Ewe verb ke}

Ameka (2019) provides a detailed analysis of the uses and meaning of the verb $k e$ 'open, open up, spread; stop, end, be finished'. For the sake of completeness and to allow for comparison with the Longgu data, we summarise that analysis here. As the glosses of the verb suggest, the verb at first sight may look to be polysemous. And, in fact, this is the stance taken by lexicographers like Westermann (1928) and Rongier (2015). However, Ameka argues for a monosemic account, and demonstrates how the various contextual interpretations can be generated from the interaction of the verb semantics with the semantics of the argument structure constructions in which it occurs as well as the semantics of the types of entities involved in the event.

The verb is primarily monovalent but has both transitive and intransitive uses. In its intransitive use, the event is construed as being internally caused. That is, the 'opening' happened not because someone did it. The verb is predicated of different classes of nominals. They can all be viewed as unitary objects which have parts. These parts could come apart along predetermined lines but without causing the disintegration of the entity into different entities. It is thus predicated of things that open naturally like séfofo 'flower' as when in full bloom; or deti 'cotton (boll)'. When a gli 'wall' or similar entity splits open by itself, the occurrence is described with the verb $k e$. The verb also collocates with the psychologised body part $\eta u$ 'eye' to express 'day break'. ${ }^{2}$ When the verb occurs with event nominals that denote situations which have a negative effect on people, such as $t s i$ 'water, rain', do 'hunger, famine' and ava 'war', it is interpreted as the situation coming to an end. Adopting a three levels of semantics approach (Wilkins and Hill 1995), Ameka (2019) explains that when the verb $k e$ is predicated of these event nominals with negative impact on people such as $d z r e$ 'quarrel' or $d z o$ 'fire' to mean 'The quarrel has ended.' or 'The fire

2 Body part terms can either refer to a physical body part, such as the physical eye, or they may refer to parts of the body where things happen because of which one feels something (inside the body). The latter are termed psychologised body parts (Ameka 2002). The same linguistic form may refer to the physical and the psychologised part: for example, Ewe $d z i$ refers to both the physical and psychologised heart. However, in some cases different words refer to the different aspects of the same part. In Ewe, for instance, the physical eye is $\eta k \dot{u}$ and the psychologised eye is $\eta u$. 
has subsided.', the same semantics represented in [A] applies. The event nominals are seen as having parts and something happens at a point in time and these parts come apart, are scattered, leading to the situation being no more. All the situations characterised by the intransitive use of the verb are irreversible. Based on the discussion so far, we propose the explication in [A] for the Ewe verb $k e$ 'open', which is a revised version of the one proposed in Ameka (2019).

\section{[A] Something $X$ ke $(X=$ flower, nut, cotton etc.)}

a. something happened to $X$ (at this time)

LEXICOSYNTACTIC

b. because of this, something happened in X in one FRAME moment, not because someone did something to it $(X)$

c. often when things like this happen, it happens like this:

before it happened:

this thing was like one thing

this thing has parts (inside it)

after it happened:

some parts move apart in places

some parts are in the same place as they

were before

this thing is like one thing

it is not two things

d. because of this, after this,

some parts are on one side,

the other parts are on the other side

e. because of this, after this,

people can see things inside this thing [people can do something with things

inside this thing, if they want] 
Furthermore, Ameka (2019) argues that the same meaning of the verb is at play when it is used transitively. In such cases, the two-place construction licenses an Effector, thus the component of 'not because someone wants it/did it' is cancelled out and the constructional meaning component of 'someone did something to something else' combines with the other components to generate the online interpretations.

In its transitive use also, there are different categories of objects that $k e$ 'open' can occur with. First are body parts that have two parts, such as $\eta k u$ 'eye', nu 'mouth', atá 'thigh', asi ' (folded) hands', glä 'jaw' and tó 'ear'. These situations can be reversed and the antonym of ke that describes it is mia 'tighten'. ${ }^{3}$ A second group is tools with parts that can be stretched out such as xéxi/sownia 'umbrella'. The spreading of other artefacts that are flexible and flat-for example, asabu 'fishing net', edo 'cloth' or tsitse 'sleeping mat' are also described with the verb ke 'open'. The reversal of this action is described by the verb yls 'fold'. Thus the opening events presented transitively with the verb $k e$ 'open' are reversible. It will be shown in the next section that the opening events described by the verb $v u$ 'open' are also reversible or their closed state can be restored by covering the opening. A further difference between the two verbs lies partly in their force dynamics (cf. Talmy 2000).

\subsection{The Ewe verb vu}

Ewe lexicographers present the verb $v u$ 'open, be open' as a polysemic word with at least five senses. Westermann (1928) gives 'open, be open' as the first sense. The verb is primarily bivalent and, in this sense, it participates in the causative/inchoative alternation as illustrated in (1), taken from Westermann (interlinear glosses added).

(1)

$\begin{array}{lll}\text { a. } & \text { vu votrú } & \text { lá } \\ \text { open door } & \text { DEF } \\ \text { 'Open the door!' } & \\ \text { b. } & \text { votrú lá } & \text { vu } \\ \text { door DEF } & \text { open } \\ & \text { 'The door opened.' }\end{array}$

3 The reversal of the opening of the body part tó 'ear' is expressed by the verb kú 'die': kú tó [die ear] 'turn a deaf ear', which refers to the ear losing its function. 
Westermann provides a further illustration of this sense with the collocation in (1c) involving an abstract object:

$\begin{array}{lll}\text { c. } & \text { vu } \quad \text { nya } \quad \text { me } \\ \text { open word containing.region } & \\ \text { 'explain, admit, confess' }\end{array}$

The focus in this chapter will be on this first sense. The other readings provided by Westermann are (ii) to move (house), leave a place, migrate, emigrate; (iii) to reach as far as, go up to/into, open on/in to; and (iv) to rise whirling, swirl up; shine; and burn. All these readings of the verb, we argue, can be motivated from the 'physical opening of entities' sense of the verb.

The verb $v u$ 'open' occurs with different types of openable objects. The first group are the containers. Containers such as adáká 'hinged box' typically have a top that when opened remains attached to the whole. Other containers have removable parts that can be lifted from the objects in order to open them. Such containers are ze 'pot', atukpá 'bottle' or nugoe 'tin'. In talking about the opening of such containers, one can either focus on the whole, as in (2a), or on the lid, (2c). One can use a dedicated construction involving a postpositional phrase where the thing that is covering the container that has to be taken apart is the dependent in such a phrase, as in (2b). In all these cases for the physical opening to occur, one does something to a part of the whole using one's hands.

(2)
a. $v u$
$z e-\varepsilon$
open
pot-DEF
'Open the pot!'

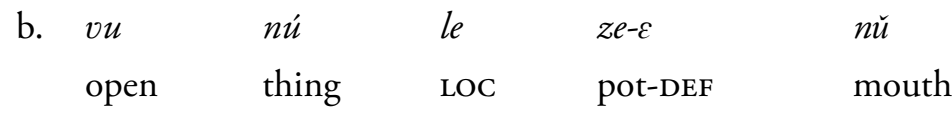
'Open the lid from the pot!'

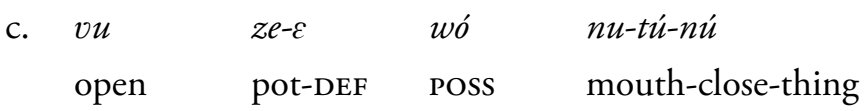
'Open the pot's lid!'

One can also refer to the content of the container when talking about the physical opening of the container. Typically this involves applying an instrument such as a tin opener or it may just involve the twisting 
of the openable part of the container. Such content can be aha 'drink', bisketi 'biscuit' or miliki 'milk', or timati 'tomato puree'. The metonymic principle here is one wants the container whose content is $\mathrm{X}$ to be opened and the container is referred to by the content. A similar metonymy is involved in talking about releasing domestic animals from their enclosed spaces such as chicken coops or sheep or goat pens. In these cases, the object of the verb can be the whole or it can refer to the content, namely, the animals.

As shown in example (1a), the verb also applies to conduits that are like parts of a whole such as $v$ (trü)/ho 'door', agbor 'gate'. The verb can also be applied to the whole entity to which the conduit gives access. For example, where one opens the door of the kitchen to gain access, one can just talk of opening the kitchen.

Similarly, the verb can be applied to the opening of institutions such as sukiu 'school' and stôs 'store'. In fact, with these nouns as object of the verb, the interpretation is vague including the physical opening of the entity (i.e. the doors) and the commencement of activities of the institution. To unambiguously express the start of an activity, a dedicated construction is employed (see example 3).

(3)

$\begin{array}{lll}\text { wo-ne-woe } & \text { wó-á-vu } & \text { stว-s } \\ \text { time-Q-time } & \text { 3PL-FUT-open } & \text { store-DEF }\end{array}$

'When will the store be opened?'

The verb can also be used to characterise the undoing of things, especially zips, buttons and hems. One can also talk about expanding or loosening a garment or a shirt by undoing the sewing using the verb. In this case, the nominal is dependent on the postposition me 'containing region'.

(4) $v u$

awu-

me

open

garment-DEF

containing region

'Open up (i.e. undo the hem of) the garment (to make it bigger)!'

The verb is also used to describe actions involving the opening of valves, pipes and taps, as well as switching on some appliances (perhaps by turning a knob) like radios and TV sets. While on a general level this use of the transitive 'open' verb in Ewe is similar to that of Longgu, in Ewe the verb is not used to talk about the turning on of lights and lamps, nor is 
it used to describe the turning on of audio recorders. It appears that Ewe categorises the turning on of appliances (radio, TV) involving turning or twisting of a knob as separate from switching on other appliances such as fans or lights that involve pressing on a button. The former is described by $v u$ 'open' while the latter are described by $s i$ 'switch on, cut (on the body)'. Actually, radios and TVs that are switched on by pressing buttons can also be described by the verb si. This further suggests that the manner in which one's hands are used to effect the change of state is a critical ingredient in the semantics of the verbs. When the verb $v u$ is used to talk about the opening of valves and taps, the object of the verb can refer to the valve or tap or to the content that is released when the valve is turned, such as water.

As noted above, the spontaneous opening of body parts is described by ke 'open'. The opening of the same parts can also be described with the verb $v u$ 'open', and in this case, the opening event is conceptualised as being purposeful and conscious. Consider the expressions in (5):

$\begin{array}{lllll}\text { vu } & \eta k u ́ l & n u / & a t a ́ l & a s i \\ \text { open } & \text { eyel } & \text { mouth/ thigh/ hand }\end{array}$

'Open your eyes/mouth/thighs/hand!'

Some components of the meaning of the verb $v u$ can be gleaned from the company it keeps as demonstrated in the preceding discussion: the objects it occurs with have parts and one does things to one of these parts leading to the part coming apart which allows one to gain access to the other parts of the entity. It was also indicated that the physical action of opening described by the verb entails the movement of the hands to bring about the change of state. We have also shown that the internal arguments can be expressed either as noun phrases (most examples) or as postpositional phrases.

When the verb enters in construction with a postpositional phrase object headed by $n u$ 'mouth' the expression gets a specialised reading, namely, 'to start or begin something'.

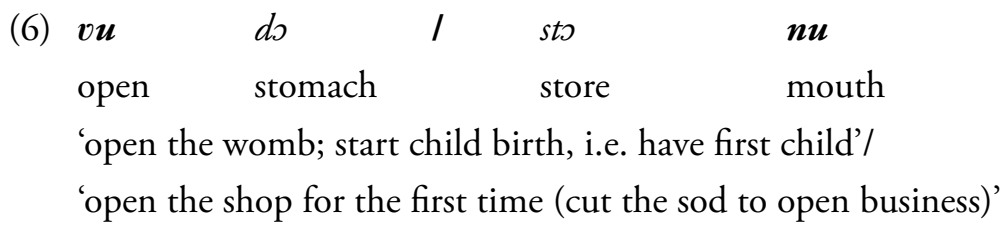


The beginning of temporal periods can also be expressed using the same structure as in (7):

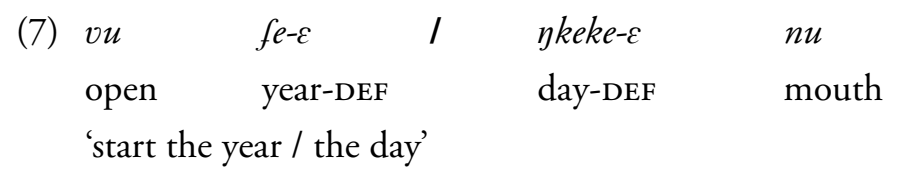

Furthermore, the verb participates in the causative/inchoative alternation as illustrated in (1). The verb $v u$ also participates in various kinds of SVCs $(8 \mathrm{~b})$ and in a resultative construction, as in (8a).
a. eho-s-wó
pétéé le
door-DEF-PL
all be.at:PRS
vu-vu ...
'All the doors are opened (but there is nobody in the house) ...'
b. devi-é
$k j \quad h \tilde{\varepsilon}$

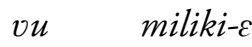
child-DEF
take knife
open milk-DEF
'The child used a knife to open the milk.'

The resultative construction involves the nominalisation of the action verb by reduplication and it is the complement of the locative verb le 'be.at:PREs'. Litivinov and Agbodjo (1988) note that the construction is not very robust in colloquial Ewe; however, an utterance such as (8a) involving the verb $v u$ is very frequent. The construction signifies a state resulting from a prior action. This result is visible. This suggests that the verb $v u$ entails a realisation of the result of the entity being opened. Based on the discussion, we propose the explication in [B] to account for the meaning of the Ewe verb $v u$.

\section{[B] Someone vu 'opened' something}

a. someone $(X)$ did something to something $(Y)$

LEXICOSYNTACTIC (at a time before now)

FRAME

b. because of this, something happened to this something $(Y)$ as this someone $(X)$ wanted it 
c. often, when someone does something like this to something, it is like this:

this something is near this someone's body

this someone thinks about this something like this:

it is (like) one thing, it has parts

someone can do something to one of these parts

when someone does this, this part can move (a little)

d. when this someone $(X)$ does this to this something $(Y)$, it happens like this:

this someone $(X)$ does something with the hands

to one part of this something $(Y)$

because of this, this someone's hand moves

for a short time as this someone wants

because of this, one part of this something

moves as this someone wants

after this, this part is no longer in the same place as before

e. because of this, after this, people can see

(POTENTIAL)

what is inside this something

To sum up, the three verbs in Ewe that carve up the sematic space of opening events have distinct semantics. Nevertheless, they may apply to the same entities, revealing different construals of the opening events, as noted for body parts. Pairs of the verbs can also occur with the same referential objects and provide different perspectives on the opening event. Thus ke and $v u$ can take agbale 'book' as complement, suggesting flipping through a book versus purposeful and targeted opening. Similarly, $k e$ and klé can take sowuia 'umbrella'. For ke it focuses on the coming apart of the blades of the umbrella while klế describes the opening wide of the umbrella. 


\section{Opening events in Longgu}

Longgu has at least 10 verbs to refer to opening events. Within this group, one verb tavangia 'open something' is semantically very similar to Ewe $v u$. It is used to refer to opening containers (e.g. a pot, a house), and is used to describe the opening of channels and conduits (Levison 1993) such as the action of turning on a tap or a radio. Like Ewe $v u$, Longgu tavangia is used when something is opened with the hands and can describe opening the whole (e.g. a house) or opening a part (e.g. door of the house). A second verb, the stative verb avure 'be open' is very similar to Ewe ke. Avure is used to refer to something that has opened naturally (e.g. flower), as well as something that is open as a result of someone doing something (e.g. an open hand, an open book). This section discusses these two verbs in detail. The discussion of avure 'be open' includes a discussion of its transitive counterpart vuresia 'open something'.

Before discussing the two verbs that are semantically similar to two of the Ewe verbs, we present an overview of the domain of 'opening' in Longgu. In addition to the verbs tavangia, avure and vuresia, Longgu has specific verbs referring to opening events in relation to body parts, such as rara'i 'open eyes'. Lexically specific verbs refer to opening events that include use of an instrument (e.g. knife, stick). While the only 'instrument' that is lexicalised in the Ewe verbs is the hands, Longgu has specialised verbs with specific instruments co-lexicalised with it. This reflects a wider pattern of lexicalisation in Longgu, where manner is encoded in many lexically specific verbs (e.g. verbs of 'carrying' (Hill 2016b; see also Heath and McPherson 2009 for a discussion of Dogon)). An example is the verb vorasia 'open out the mat/umbrella/hand', which reflects the manner of opening.

\subsection{The Longgu verbs avure 'be open' and vuresia 'open something'}

The first two verbs to be discussed are morphologically related: avure 'be open', and vuresia 'open something'. The intransitive verb avure 'be open' is formed by a stative prefix $a$ - and the verb root vure-. In Longgu the stative prefix $m a-$, of which $a$-is a variant, is not productive. 
The verb avure 'be open' is used for things made of leaf or cloth, as well as an open hand, and an open book. It is used to describe the open bud in a flower, which occurs naturally, and to opened food parcels, which are wrapped in leaves. It is notable that avure is used to describe an open hand as the transitive counterpart is vorasia 'open out, unfold' and not vuresia 'open something'.

Longgu avure refers to the result of a person's action (e.g. opening the hand or opening a book) as well as a naturally occurring action, such as the opening of a flower. In this respect, Longgu avure is similar to Ewe ke and the English verb 'open', which can refer to something independently open, i.e. in the condition of being open (e.g. the hand is open), it can open on its own, or it can be opened by someone (Talmy 2000: 85). Longgu differs from English in that it distinguishes the kind of object that is being opened (e.g. the intransitive form tavatavangi 'be open' refers to an open door, while avure refers to an open flower or food parcel). (See section 3.2 for a discussion of tavangia 'open something'.)

The transitive form vuresia 'open something' is used to describe the same opening events that have been discussed for avure (with the exception of opening the hands). The action of opening is done with the hands. What seems to be essential here is that it is used with an object that 'separates along predictable lines' (Bowerman 2005) (as opposed to being used with an object that has visible parts). Longgu consultants noted that this verb is used when you want to see something inside. Whether to see something or do something with what is inside, this opening action 'affords access to something' (Bowerman 2005).

The verb vuresia 'open something' can describe the opening event involved in opening a food parcel — the cooked food within the parcel covered in leaves is opened by removing the outer covering (the leaves), giving access to the food inside. Longgu vuresia allows the object to be the container (the food parcel) or the food within it.
a. $a m i$
vure-si-i
buta-gi
IPL.EXCL.SBJ
open-TRS-3PL.INAN
food parcel-PL
'We opened the food parcels.'
b. ami
IPL.EXCL.SBJ

$$
\text { vure-si-i }
$$$$
\text { open-TRS-3PL.INAN food-PL }
$$
'We opened the food (i.e. removed the leaves from the cooked food).' 
The stative verb avure 'be open' is similar to Ewe $k e$ (in its intransitive use) in that it can refer to something that opens naturally, such as a flower, but different from it in that it can refer to something that has been opened by someone (e.g. a food parcel, book or hand). Like the Ewe ke, it is not used for objects such as doors, and pots, which have parts that can be separated and where the action can be reversed. Longgu avure 'be open' is vaguer than Ewe ke in delineating the kind of object that can 'be open', but it does not refer to opening objects that have clearly identifiable parts that can be removed from the whole object. While we can identify, for example, the outer part of a food parcel, the pages of a book and the fingers or palm of a hand, the object is perceived as a whole object rather than an 'object with parts' although there are predictable ways in which the object opens (see explication of avure in [C] below). A difference between intransitive Ewe ke and Longgu avure is in the other objects which it can be predicated of. Thus, Ewe ke, as noted, occurs with 'eye' to express 'day break' and with other entities as subject to yield a reading of 'stop, finish'.

\section{[C] Something $X$ avure $(X=$ flower, food parcel, book, hand)}

a. something happened to $X$ (at this time)

LEXICOSYNTACTIC

b. because of this, something happened in $X$ in one

FRAME moment

c. often when things like this happen,

PROTOTYPICAL it happens like this:

before it happened:

this thing was like one thing

after it happened:

some parts are not in the same place as they were before

some parts are in the same place as they

were before

this thing is like one thing

it is not like two things

e. because of this, after this, 
f. because of this, after this,

people can see things inside this thing

[people can do something with things inside this thing, if they want]

The transitive verb vuresia 'open something' is similar to the transitive use of Ewe ke in the objects that it can apply to: body parts, tools such as books and umbrellas, and it can also be used with soft and flexible objects such as cloth or leaves. A crucial difference between the two is that for vuresia opening events are done with the hands while for the transitive $k e$ opening events need not involve the hands. The other Ewe verbs $v u$ and klế do entail the use of the hands just as vuresia and tavangia do.

\subsection{The Longgu verb tavangia 'open something'}

Among Longgu verbs of 'opening', the transitive verb tavangia occurs most frequently in texts and combines productively with other verb forms as a serial verb or compound form. Tavangia is used to refer to opening containers, channels and paths (Levison 1993). Tavangia consists of the root tava, the transitive suffix (in this case -ngi; other consonants are also found in the transitive suffix e.g. the si of vuresia) and a pronominal object suffix $-a$ (third person singular object suffix).

Tavangia 'open something' is used when the action of opening involves the hands (or body) and when the object being opened is one that can be described as having parts. It is used to describe the opening of 'containers', such as a house or a pot. Tavangia can refer to opening the whole container (e.g. the house) or part of the container (e.g. door of the house), as exemplified below.

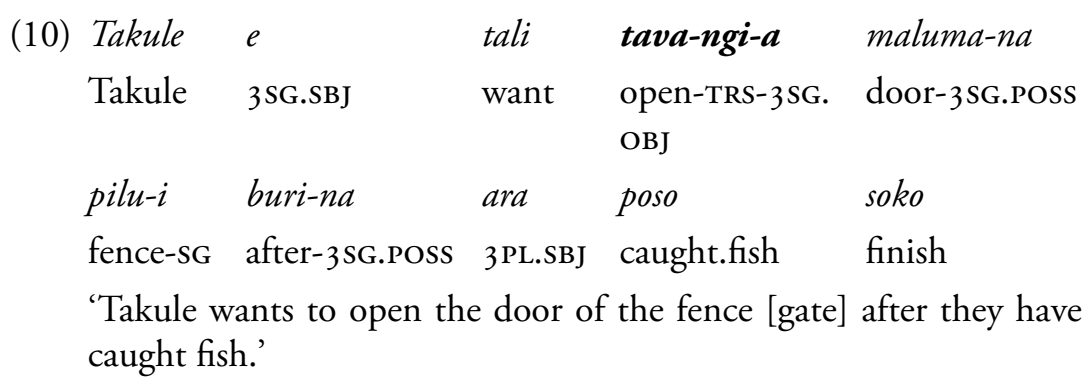



(11) La mai m-e
tava-ngi-a luma girua-i
go hither CONJ-3SG.SBJ
open-TRS-3SG. house 3DU.POSS-SG OBJ
'[He] came and he opened their (two) house.'

The verb can form part of an SVC, as shown in example (12), where the verb oli 'do again, return' precedes the object pronoun ( $-a$ '3SG.OBJ').

(12)
Christopher Kaimali $e$
tava-ngi-oli-a
dingidingi.
Christopher Kaimali 3SG.SBJ open-TRS-again-3SG.OBJ door.

'Christopher Kaimali opened the door again.'

An intransitive verb is formed by reduplicating the root. The object suffix is omitted, tavatavangi:

$\begin{array}{lll}\text { tapwi } & e & \text { tavatavangi } \\ \text { tap } & \text { 3SG.SBJ } & \text { open }\end{array}$

'The tap is on/open.'

Note that there are two possible antonyms for tavatavangi 'be open/on'. The first is dingidingi 'be closed' (a reduplication of dingia 'close something'), which also has a nominal meaning of 'door' (see example (12)).

(14)

$\begin{array}{lll}\text { tapwi } & e & \text { dingidingi } \\ \text { tap } & \text { 3SG.SBJ } & \text { closed }\end{array}$

'The tap is off.'

The alternative antonym is bono 'be blocked', which, if used in the context of the tap, could only mean the tap is blocked, not 'off. Similarly, if the door is closed it is dingidingi 'be closed', and if it is blocked it is bono 'blocked'. The use of the antonym bono reflects the meaning of tavangia to express open channels and paths (i.e. a path can be blocked).

Tavangia 'open something' is also used to refer to turning on audio recorders and lights or lamps, expressing that the object is a conduit or channel for something, such as sound or light. The notion of 'path' or 'channel' as senses expressed by tavangia 'open something' is also found in compound verbs formed with tava. 
(15) a. bou-tava

head-open

'enter; go through; e.g. bush, cloud, village'

b. isi-tava

bound morpheme-open

'to go outside from inside' (e.g. to out from the house)

c. ave-tava

bound morpheme (flood)-open

'to be at low tide; ebb'

The semantic explication of tavangia 'open something' is given in [D]:

\section{[D] Someone tavangia 'opened' something}

a. someone $(X)$ did something to something $(Y)$

LEXICOSYNTACTIC

(at a time before now)

FRAME

b. because of this, something happened to this something $(Y)$ as this someone wanted it

c. often, when someone does something like this to something,

PROTOTYPICAL it is like this:

this something is near this someone's body

this someone thinks about this something like this:

it is (like) one thing, it has parts

someone can do something to one of these parts

when someone does this, this part can move (a little)

d. when this someone $(X)$ does this to this something $(Y)$, it happens like this:

this someone $(X)$ does something with the hands to one part of this something $(Y)$

because of this, this someone's hand moves for a short time as this someone wants 
because of this, one part of this something moves as this someone wants

after this, this part is no longer in the same place as before

e. because of this, after this, people can see

what is inside this something

something/someone can move from one place

to another after this

\section{Explications and discussion of $v u$ and tavangia, and ke and avure}

The discussion so far has shown that while Ewe and Longgu differ in the lexical density in the domain of verbs of opening events, there are several verbs in each language that express similar meanings. At the same time, the languages vary in the range of openable objects that are used with these verbs. In this section we propose shared explications for Ewe ke and Longgu avure (in [E]) and for Ewe vu and Longgu tavangia (in [F]). The explications proposed for ke and avure is an improved version of the one presented in Ameka (2019) for ke. We highlight where the differences between the languages lie in specific components.

\section{[E] Something $X$ ke $(X=$ flower, nut, cotton etc.), something $X$ avure $(X=$ flower, food parcel, book, hand)}

\section{Ewe}

something happened to $X$ (at this time)

because of this, something happened in $\mathrm{X}$ in one moment

not because someone did something to it $(X)$

\section{Longgu}

something happened to $X$ (at this time)

because of this, something happened in X in one moment

\section{Semantic template}

LEXICOSYNTACTIC FRAME 
often when things like this happen,

it happens like this:

before it happened:

this thing was like one thing

this thing has parts

(inside it)

after it happened:

some parts move apart

in places

some parts are in the same place as they were before

this thing is like one thing

it is not two things often when things like this happen,

it happens like this:

before it happened:

this thing was like

one thing

after it happened:

some parts are not in

the same place as they

were before

some parts are in the same place as they

were before

this thing is like one

thing

it is not like two things

because of this, after this, because of this, after this,

some parts are on one

side, the other parts are

on the other side

because of this, after this,

people can see things

inside this thing

[people can do something

with things

inside this thing, if they

want] people can see all sides

of this thing
PROTOTYPICAL

SCENARIO

EFFECT

(POTENTIAL)

OUTCOME

The explication in [E] shows that the lexicosyntactic frame for ke and avure is shared, except that in Longgu something can be avure 'be open' both naturally and because someone did something, whereas intransitive ke is only used when the opening event happened naturally. For this reason, only Ewe includes the component 'not because someone did something to it $(\mathrm{X})^{\prime}$. A key difference between the two languages lies in the prototypical scenario. Ewe views the openable object as having parts (this thing was like one thing, this thing has parts (inside it)), whereas for Longgu it is 
important that 'this thing was like one thing', but there is no mention of 'parts'. For both Ewe and Longgu the Effect and (Potential) Outcome are the same, or may be the same. That is, ke and avure are both verbs that express opening events (effect and outcome) but they differ in terms of which openable objects these verbs are used with.

Based on the discussion of the verbs so far, we propose explication $[\mathrm{F}]$ for the verbs $v u$ and tavangia to account for all the uses related to the physical action of opening an entity. The explication in $[\mathrm{F}]$ shows that Ewe and Longgu share almost all of the semantic components, with the exception of the (Potential) Outcome.

The difference in the potential outcome accounts for the use of tavangia in Longgu to express 'paths and channels' (e.g. see the compound verbs listed in example (15)), and the discussion of tavangia as a verb to describe the 'opening' or turning on of lamps, lights, audio recorders, as well as opening a container (e.g. a door of a house). By contrast, Ewe $v u$ is used with containers, and is more limited in its use to express paths and channels. The close semantic similarity between these two verbs suggests that the sense of 'opening' a container is cross-linguistically common. Not surprisingly, the use of verbs of 'opening' to express opening a meeting or opening a channel (e.g. sound, light) is more specific to a particular language.

\section{[F] Someone vu 'opened' something, someone tavangia 'opened' something}

Someone $(X)$ did something to something $(Y)$

\section{Ewe}

someone $(X)$ did something to something $(Y)$ (at a time before now)

because of this, something happened to this something $(Y)$ as this someone $(X)$ wanted it

\section{Longgu}

someone $(X)$ did something to something $(Y)$ (at a time before now)

because of this, something happened to this something $(Y)$ as this someone wanted it

\section{Semantic template}

LEXICOSYNTACTIC FRAME 
often, when someone does something like this to something, it is like this:

this something is near this someone's body

this someone thinks about this something like this:

it is (like) one thing, it has parts

someone can do something to one of these parts

when someone does this, this part can move (a little)

when this someone $(X)$ does this to this something $(Y)$, it happens like this:

this someone $(X)$ does something with the hands to one part of this something $(Y)$

because of this, this someone's hand moves for a short time as this someone wants

because of this, one part of this something moves as this someone wants after this, this part is no longer in the same place as before

because of this, after this, people can see what is inside this something often, when someone

does something like this to

something, it is like this:

this something is near this someone's body

this someone thinks about this something like this:

it is (like) one thing, it has parts

someone can do something to one of these parts when someone does this, this part can move (a little)

when this someone $(X)$ does this to this something $(Y)$, it happens like this:

this someone $(X)$ does something with the hands to one part of this something $(Y)$

because of this, this someone's hand moves for a short time as this someone wants

because of this, one part of this something moves as this someone wants after this, this part is no longer in the same place as before

because of this, after this,

POTENTIAL OUTCOME this something something/someone can move from one place to another after this 


\subsection{Summary of the semantics of 'opening' events in Ewe and Longgu}

A comparison of the semantics of verbs of 'opening' in Ewe and Longgu has provided further examples of languages that carve up the semantic space of 'opening' events differently. Where Ewe has three verbs, Longgu has over 10. Longgu has specific verbs to refer to opening of body parts, and specific verbs that refer to opening specific objects with instruments. At the same time, both Ewe and Longgu have verbs whose major senses involve the characteristics suggested for opening events in English: (a) a unitary object, although it may have parts such as a pot with a lid; (b) an object that separates along predetermined lines not unpredictably (hence actions of opening are usually reversible; objects that can be opened can also be closed); and (c) separation affords access to something (e.g. a content, an interior space or a previously concealed part of the object with which you can do something). In Longgu, opening events involve separation that affords access to something but it distinguishes opening events that involve objects that have clear parts (i.e. tavangia 'open' e.g. the door), and opening events that involve objects that can be separated along predetermined lines, but which do not necessarily have clearly identifiable parts (i.e. vuresia 'open' e.g. the food parcel/food). Associated with this is the outcome of opening the openable objectfor the objects that have parts, this typically allows something to move through the object (e.g. a person through a door, water through a pipe, sound through a radio), whereas for the objects that do not clearly have parts, the action may simply allow someone to see something inside or do something with part of the object (e.g. food in the parcel).

One cultural object of Longgu that consists, in part, of leaves and can also be viewed as having parts is an $u m u$ 'stone oven'. An $u m u$ is a place to cook food in the ground. The food is cooked by placing it on hot stones and covering the food with more hot stones, old leaves and cloth such as old hessian bags. The umu 'stone oven' can be partially opened, i.e. to check the food, or it can be completely uncovered to remove the food. Both verbs (tavangia and vuresia) can be used to refer to opening a stone oven. The use of the verbs depends on whether the speaker is perceiving the oven as having 'parts' and doing something with the food (e.g. removing it from the oven), in which case the verb tavangia 'open something' is used, or whether the speaker is checking the food, in which case the top leaves and hessian may be removed and the verb vuresia 'open something' 
is used. Speakers describe the purpose of opening the oven, like opening the food parcels made of leaf, to see what is inside and to do something (e.g. eat the food from the food parcel, check when the food in the oven is ready). This analysis is supported by the antonym of vuresia, referring to opening an oven, which is kuvia 'cover it'.

The key difference between tavangia and vuresia, we suggest, is that while tavangia is used when the object has visible 'parts', and these parts show where the object can be separated (e.g. the lid of a pot, the door of a house), vuresia is used when the object can be separated along predictable lines (e.g. the leaves of a book, the cooked leaves covering food) but the object does not need to be perceived as having separate parts. For example, a woven basket can be 'undone' using the verb vuresia 'open something'. Note Ewe uses the verb $v u$ for undoing buttons, hems and zips. The basket is made of leaves, and in addition it is not perceived of as having clearly separate parts. This analysis may also explain why 'moving through' something isn't part of the meaning of vuresia.

Ewe distinguishes opening events according to whether the opening event is natural or caused ( $k e$ and $v u$ ) and according to whether the opened object has an 'inside part and outside part' (klế), and according to whether the action is reversible or irreversible (the natural opening events of, for example, flowers or nuts are irreversible). Longgu does not pay attention to whether the opening event is natural or caused, but does pay attention to whether the action is reversible or irreversible (underlying this seems to be whether the object is made up of clear 'parts' or whether there is separation along predictable lines) and does pay attention to the kind of object being opened (and therefore the manner). In both languages, more than one verb can be used for the same opening event, but the perception is different. For example, in Ewe a different verb is used depending on whether something is perceived as opening naturally or is caused to be open (e.g. body parts), and in Longgu a different verb is used depending on whether something is perceived as having clear parts or just predictable lines of opening (e.g. a stone oven). We have shown that the Ewe verbs have some interpretations that are extensions of the physical action of opening something. Thus, $k e$ in its internally caused and intransitive use is extended to talking about the ending of negative impact events. On the other hand, the verb $v u$, in specialised construction with postposition $n u$ 'mouth', is used to talk about the commencement of situations or of 
temporal periods. In the latter case, Ewe is similar to English where the English open is also used to talk about the start of events-for example, to open the meeting (see Levison 1993).

The comparison between verbs of opening in Ewe and Longgu has highlighted the complexity of verbs of opening cross-linguistically, using NSM to show where meaning differences between the two languages lie in a small number of semantically similar verbs.

The human experience of opening objects is both a universal experience and one that may reflect a specific culture, as both material culture and different ways of perceiving and construing objects are involved.

\section{References}

Ameka, Felix K. (2002). Cultural scripting of body parts for emotions: On 'jealousy' and related emotions in Ewe. Pragmatics \& Cognition 10 (1): 27-55.

Ameka, Felix K. (2017). Meaning between algebra and culture: Auto-antonyms in the Ewe verb lexicon. In Lisa L.S. Cheng, Maarten Hijzelendoorn, Hilke Reckman and Rint Sybesma (eds), Crossroads Semantics: Computation, Experiment and Grammar, Amsterdam: John Benjamins, 227-48. doi.org/ 10.1075/z.210.14ame.

Ameka, Felix K. (2019). "The Nut opens" and "Hunger ends": Verb constructions at the syntax-semantics interface. In James Essegbey, Dalina Kallulli and Adams Bodomo (eds), The Grammar of Verbs and their Arguments: A CrossLinguistic Perspective. Cologne: Rüdiger Köppe, 59-84.

Ameka, Felix K. and James Essegbey (2007). 'CUT and BREAK' verbs and the causative/inchoative alternation in Ewe. Cognitive Linguistics 18 (2): 241-50. doi.org/10.1515/cog.2007.012.

Bowerman, Melissa (2005). Why can't you 'open' a nut, or 'break' a cooked noodle? Learning covert object categories in action word meanings. In Lisa GershkoffStowe and David H. Rakison (eds), Building object categories in developmental time. Mahwah, NJ and London: Lawrence Erhlbaum Associates, 209-43.

Fillmore, Charles J. and Beryl T. Atkins (1992). Toward a frame-based lexicon: The semantics of RISK and its neighbors. In Adrienne Lehrer, Eva Feder Kittay and Richard Lehrer (eds), Frames, Fields, and Contrasts: New Essays in Semantic and Lexical Organization, Albany: Lawrence Earlbaum and Associates, 75-102. 
Goddard, Cliff and Anna Wierzbicka (2004). Cultural scripts: What are they and what are they good for? Intercultural Pragmatics 1 (2): 153-66. doi.org/ 10.1515/iprg.2004.1.2.153.

Goddard, Cliff and Anna Wierzbicka (2009). Contrastive semantics of physical activity verbs: 'Cutting' and 'chopping' in English, Polish, and Japanese. Language Sciences 31: 60-96. doi.org/10.1016/j.langsci.2007.10.002.

Goddard, Cliff and Anna Wierzbicka (2016). Explicating the English lexicon of 'doing' and 'happening'. Functions of Language 23 (2): 214-56. doi.org/ 10.1075/fol.23.2.03god.

Heath, Jeffrey and Laura MacPherson (2009). Cognitive set and lexicalization strategy in Dogon action verbs. Anthropological Linguistics 51 (1): 38-63. doi.org/10.1353/anl.0.0001.

Hill, Deborah (2016a). Narrative texts and clause order: Changes over time. Australian Journal of Linguistics 36 (3): 1-19. doi.org/10.1080/07268602. 2015.1134299.

Hill, Deborah (2016b). Bride-price, baskets, and the semantic domain of 'carrying' in a matrilineal society. Oceanic Linguistics 55 (2): 500-52. doi.org/10.1353/ ol.2016.0023.

Kecskes, Istvan (2008). Dueling contexts: A dynamic model of meaning. Journal of Pragmatics 40 (3): 385-406. doi.org/10.1016/j.pragma.2007.12.004.

Levinson, Stephen C. (2000). Presumptive Meanings: The Theory of Generalized Conversational Implicature. Cambridge, MA, and London: MIT Press. doi.org/10.7551/mitpress/5526.001.0001.

Levison, Libby (1993). The topic is open. Working paper presented at the University of Pennsylvania Linguistics Forum 1993. Available at: repository. upenn.edu/hms/98.

Litvinov, Victor, and Kofi Agbodjo (1988 [1983]). Resultative in Ewe. In Vladimir P. Nedjalkov (ed.), Typology of Resultative Constructions: Translated from the Original Russian Edition, Amsterdam: John Benjamins, 231-37. doi.org/ 10.1075/tsl.12.19lit.

Majid, Asifa, Melissa Bowerman, Miriam Van Staden and James S. Boster (2007). The semantic categories of cutting and breaking events: A crosslinguistic perspective. Cognitive Linguistics 18 (2): 133-52. doi.org/10.1515/cog.2 007.005 .

Rongier, Jacques (2015). Dictionaire éwé-français. Paris: L’Harmattan. 
Talmy, Leonard (2000). Lexicalization patterns. In Toward a Cognitive Semantics. Volume II: Typology and Process in Concept Structuring. Chapter 1. Cambridge, MA: Massachusetts Institute of Technology. doi.org/10.7551/ mitpress/6848.003.0003.

Westermann, Diedrich (1928). Evefiala: Ewe-English Dictionary. Berlin: Dietrich Reimer.

Wilkins, David and Deborah Hill (1995). When GO means COME: Questioning the basicness of basic motion verbs. Cognitive Linguistics 6 (2-3): 209-59. doi.org/10.1515/cogl.1995.6.2-3.209. 
This text is taken from Meaning, Life and Culture: In conversation with Anna Wierzbicka, edited by Helen Bromhead and Zhengdao Ye, published 2020 by ANU Press, The Australian National University,

Canberra, Australia.

doi.org/10.22459/MLC.2020.02 